\title{
ORIGINAL ARTICLE Effect of nasal continuous positive airway pressure on the pharyngeal swallow in neonates
}

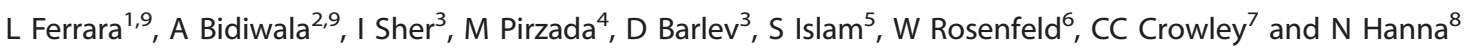

OBJECTIVE: Feeding neonates orally while on nasal continuous positive airway pressure (nCPAP) is a common practice. We hypothesize that pressurized airflow provided by nCPAP will alter the swallowing mechanism in neonates, increasing the risk of aspiration during oral feeding.

STUDY DESIGN: Infants receiving nCPAP with a RAM cannula and tolerating at least $50 \%$ of their feeding orally were included in the study (one term; six preterm infants). Each participant underwent a videofluoroscopic swallow study while on nCPAP and off nCPAP. A non-parametric signed-rank test was used for paired data.

RESULT: The incidence of deep penetration $(P=0.03)$ and aspiration $(P=0.01)$ decreased significantly off-nCPAP compared with onnCPAP. However, the incidence of mild penetration $(P=0.65)$ and nasopharyngeal reflux $(P=0.87)$ remained the same under both conditions.

CONCLUSION: Oral feeding while on-nCPAP significantly increases the risk of laryngeal penetration and tracheal aspiration events. We recommend caution when initiating oral feedings on nCPAP.

Journal of Perinatology (2017) 37, 398-403; doi:10.1038/jp.2016.229; published online 5 January 2017

\section{INTRODUCTION}

Preterm and term infants with cardio-respiratory illness frequently receive ventilatory assistance. Nasal continuous positive airway pressure (nCPAP) is a proven mode of noninvasive ventilatory support in neonates, as it delivers positive inflation pressure to the airway throughout the respiratory cycle. ${ }^{1,2}$ Oral feeding of neonates while on nCPAP is a common practice in many neonatal intensive care units (NICUs); however, the effect of this practice on the neonatal swallowing mechanism has never been investigated.

A close inter-relationship between swallowing and breathing exists, leading to some controversy regarding the safety of starting oral feeding while infants are on nCPAP. No reports on the effect of nCPAP on infant swallowing were found on a literature search; however, nCPAP is reported to induce dilatation of the laryngeal opening in preterm infants ${ }^{3}$ and inhibits the swallow reflex in adults. 4

For preterm infants, some neonatologists advocate initiation of oral feeding at 34 weeks of gestational age, even if they require nCPAP. Others strictly wait for nCPAP to be discontinued before oral feeding is established, fearing that infants on nCPAP may have difficulty coordinating breathing and swallowing, resulting in airway compromise..$^{5-7}$ For term infants, many propose that oral feedings can be established once respiratory stability is demonstrated, either with or without nCPAP. Such controversies exist owing to lack of evidence on the effect(s) of nCPAP on swallowing in human neonates.

This study was designed to assess the effects of nCPAP on pharyngeal swallowing in neonates. We hypothesized that nCPAP will increase the risk for airway compromise and aspiration during oral feeding in neonates.

\section{MATERIALS AND METHODS}

\section{Participants}

This study was approved by the hospital institutional review board, and informed written consent was obtained from both of the parents of each participant before enrollment. All participants received care in the NICU at Winthrop University Hospital. Inclusion criteria included infants with a postmenstrual age (PMA) of $\geqslant 34$ weeks, those who received nCPAP through a RAM cannula device and those who tolerated at least $50 \%$ of their total fluid intake by mouth from a bottle, as determined by their medical team. None of the infants enrolled in this study demonstrated clinical signs of feeding intolerance. Infants with other comorbidities, such as upper airway anomalies, brain injury, neuromuscular disease and lifethreatening congenital disease, were excluded from this study. An original enrollment goal of 15 infants was approved by the hospital institutional review board; however, after the interim analysis of the first seven patients (as reported in this article), our NICU Safety Committee decided to halt further enrollment of patients as it was clear that placing infants on nCPAP while feeding them orally significantly increases the risk of airway compromise and aspiration. Therefore, it was unethical to continue exposing infants to such risks.

Demographic information on the subject (such as gender, race, gestational age at birth, birth weight, PMA and day of life at the time of the study) and clinical information (such as feeding milestones, medical diagnoses and respiratory status) were routinely recorded on a data collection form. At the time of data collection, each participant was receiving nCPAP of $5 \mathrm{~cm} \mathrm{H}_{2} \mathrm{O}$ and mean $\mathrm{FiO}_{2} 0.25$ (range of $0.21-0.37$ ). At the time of enrollment, all infants were on a RAM cannula (Neotech,

\footnotetext{
${ }^{1}$ Division of Pediatric Gastroenterology, Children's Medical Center at Winthrop University Hospital, Mineola, NY, USA; ${ }^{2}$ Division of Respiratory and Sleep Medicine, Children's

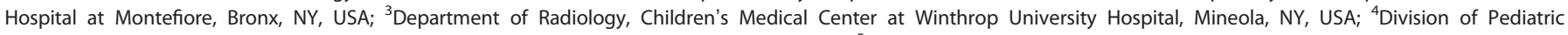

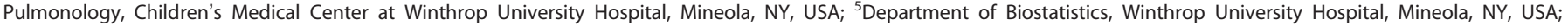

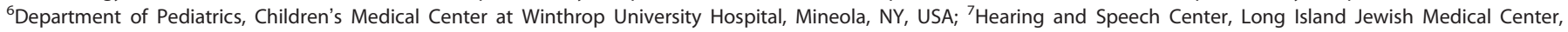

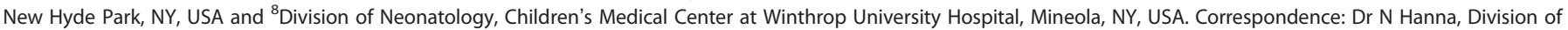
Neonatology, Children's Medical Center at Winthrop University Hospital, 259 1st Street Mineola, NY 11501, USA.

E-mail: NHanna@winthrop.org

${ }^{9}$ These authors contributed equally to this work.

Received 26 September 2016; accepted 18 October 2016; published online 5 January 2017
} 
Valencia, CA, USA), with binasal prongs secured in place with steri-tape and connected to ventilator-derived CPAP machine (Drager Medical, Cubeck, Germany) to provide constant flow. In our NICU, a RAM cannula is used as the primary nasal interface to deliver CPAP for infants on $\mathrm{FiO}_{2}$ of $\leqslant 0.40$.

Nasal noninvasive ventilation can be delivered by a variety of devices that generate either a preset flow or a preset pressure to the nares. ${ }^{8}$ Conventional ventilators are used in pressure control mode, which allow for compensation of the expected leaks when using noninvasive ventilation interfaces such as CPAP prongs or RAM cannulas. ${ }^{8}$ It was reported that the RAM device has good pressure transmission when the nasal cannula is properly sized with the transmission of $60-70 \%$ of the set peak airway pressure and positive end-expiratory pressure ${ }^{8}$ that is similar to other commonly used nCPAP devices. ${ }^{9}$

\section{Procedure}

Video fluoroscopic swallow studies (VFSS) were utilized to identify changes in the pharyngeal phase of swallowing in infants while receiving nCPAP (on nCPAP) as compared with without nCPAP (off nCPAP). Once consented, each participant was not fed for $3 \mathrm{~h}$ prior to the scheduled VFSS time. A neonatal nurse, neonatal respiratory therapist and the research team (physician and swallowing specialist) accompanied each patient from the NICU to the radiology suite. Each infant was transported in a mobile isolette while remaining on nCPAP and a pulse oximeter (Masimo Corporation, Irvine, CA, USA). The radiology table was placed in a vertical position. Each infant was swaddled and placed in a sitting position in a Tumble Form Infant seat (J.A. Preston, Jackson, MI, USA) attached to a MAMA System (MAMA Sytems, Oconomowoc, WI, USA) in a $45-90^{\circ}$ angled position. Participants were protected against radiation exposure by the placement of a small lead shield over their pelvic area. The pulse oximeter remained in use for the entirety of the evaluation.

Once participants were positioned, they were first offered a bottle of their typical feeding (infant formula or expressed breast milk) for $90 \mathrm{~s}$. All infants were fed by a single feeding and swallowing specialist. Then the pediatric radiologist positioned the camera head of the video fluoroscopy machine to obtain lateral laryngeal view and narrowed the field of view to reduce radiation exposure. Fluoroscopy was set to 30 frames per second and turned on only during the time of the barium swallow to reduce radiation exposure. All infants included in the study were receiving nCPAP via RAM cannula as part of their routine medical care before enrollment and underwent a VFSS while receiving nCPAP via RAM cannula. Each participant was fed room temperature thin liquid barium (Varibar Thin Liquid Barium Sulfate for Suspension; Lake Success, NY, USA) from a $60 \mathrm{ml}$ Similac Volu-Feeder (Columbus, OH, USA) with an attached Similac Infant Nipple and Ring (standard flow) (Columbus, OH, USA); a total of 20 swallows were recorded. These swallows were termed 'on-nCPAP' swallows. As per the protocol defined by Fink and Ross, ${ }^{10}$ the barium was diluted by $50 \%$ with water to have a viscosity most similar to human milk or formula.

The swallows were initially assessed in real time for any swallowing dysfunction. All seven participants were observed to have abnormalities in their swallowing function; therefore, we repeated the VFSS assessment with the nCPAP turned off and replaced by nasal cannula (1 liter/min) to deliver oxygen similar to the amount given by nCPAP. These swallows were termed 'off-nCPAP' swallows. Radiation exposure, per participant, was limited to a maximum exposure time of $1 \mathrm{~min}$.

Both data sets, 'on-nCPAP' and 'off-nCPAP', were captured using Philips' Easy Diagnostic digital fluoro radiographic unit (Phillips Medical Systems,
Shelton, $\mathrm{CT}$, USA) and simultaneously recorded and saved electronically onto the TIMS DICOM system (Foresight Imaging, Chelmsford, MA, USA). All data points were then transferred onto a DVD and immediately labeled with a unique identifier with no link to identifying or protected information. DVDs and the original copies of the consent were kept in a locked file cabinet.

\section{Study definitions}

Swallows in the on-nCPAP and off-nCPAP conditions were assessed in slow motion and with a frame-by-frame analysis (30 frames per second) using the TIMS system. Every swallow of liquid was analyzed for the following variables:

1. Laryngeal penetration, defined by the occurrence of barium underneath the epiglottis within the laryngeal vestibule. Mild laryngeal penetration, defined by the occurrence of barium remaining in the upper third quadrant of the laryngeal vestibule. Deep laryngeal penetration, defined by the occurrence of barium in the laryngeal vestibule to the level of the vocal folds. ${ }^{11-13}$

2. Tracheal aspiration, defined by the occurrence of barium below the level of the true vocal cords. ${ }^{11,14,15}$

3. Nasopharyngeal reflux, defined by the occurrence of barium detected in the nasopharynx, posterior or superior to the velum. ${ }^{11}$

4. Pharyngeal residue, defined by the presence of residual barium coating the pharyngeal walls, pooling in the vallecula or pyriform sinuses postswallow (absent/mild/severe).

5. Occurrence of a cough during aspiration (present/absent).

6. Ability to clear the laryngeal vestibule during penetration events (sufficient/insufficient).

7. Ability to clear the trachea during aspiration events (sufficient/ insufficient).

\section{Statistical analysis}

Statistical analysis was performed using SAS 9.4 (SAS Institute, Carey, NC, USA). Descriptive statistics were presented using the mean \pm s.d. and median (first quartile-third quartile). Ninety-five percent confidence intervals were calculated for means of off-nCPAP versus on-nCPAP differences. Off-nCPAP and on-nCPAP values were compared using a non-parametric signed-rank test for paired data. Statistical significance was set at $P<0.05$.

Interjudge reliability was assessed, and all seven VFSS videos were reassessed by an independent clinician who was blinded to the identity of participants, the analysis results and the hypothesis of the research study.

\section{RESULTS}

As seen in Table 1, six of the seven study participants were born preterm (mean gestational age at birth of 28 weeks and 3 days) and one was born at term. Participants' mean day of life and PMA at the time of data collection were 68 days and 37 weeks, respectively. None of the infants enrolled in this study demonstrated clinical signs of feeding intolerance. In addition, the medical staff did not report oral feeding difficulties (coughing, spitting or regurgitation) for any of the enrolled participants.

Table 1. Participants' demographics $(n=7)$

\begin{tabular}{|c|c|c|c|c|c|c|}
\hline 1 & Female & Caucasian & 38.4 & 2.6 & 11 & 40.1 \\
\hline 2 & Male & African American & 30.2 & 0.8 & 91 & 43.2 \\
\hline 4 & Female & Caucasian & 26.2 & 0.8 & 78 & 37.3 \\
\hline 5 & Male & Caucasian & 24.5 & 0.6 & 89 & 37.3 \\
\hline 6 & Male & African American & 25.0 & 0.6 & 64 & 34.1 \\
\hline 7 & Male & Caucasian & 27.4 & 1.1 & 74 & 38.1 \\
\hline
\end{tabular}


Table 2. Percentage of all swallows identified with swallowing dysfunction

\begin{tabular}{|c|c|c|c|c|c|}
\hline \multirow[b]{2}{*}{ Variable } & \multicolumn{2}{|c|}{ on-nCPAP } & \multicolumn{2}{|c|}{ off-nCPAP } & \multirow[b]{2}{*}{ Signed-rank P-value } \\
\hline & Mean \pm s.d. & Median (q1-q3) & Mean \pm s.d. & Median (q1-q3) & \\
\hline Deep pen. \% & $43.7 \pm 15.4$ & $38.5(30-59)$ & $25.3 \pm 8.8$ & $25(18.2-32)$ & 0.031 \\
\hline Aspiration \% & $33.5 \pm 9.4$ & $30(27.3-44.4)$ & $14.6 \pm 7$ & $15(9.1-20)$ & 0.016 \\
\hline Naso. \% & $42.8 \pm 48.5$ & $18.2(0-100)$ & $44.2 \pm 45.4$ & $18.2(5-92)$ & 0.875 \\
\hline
\end{tabular}

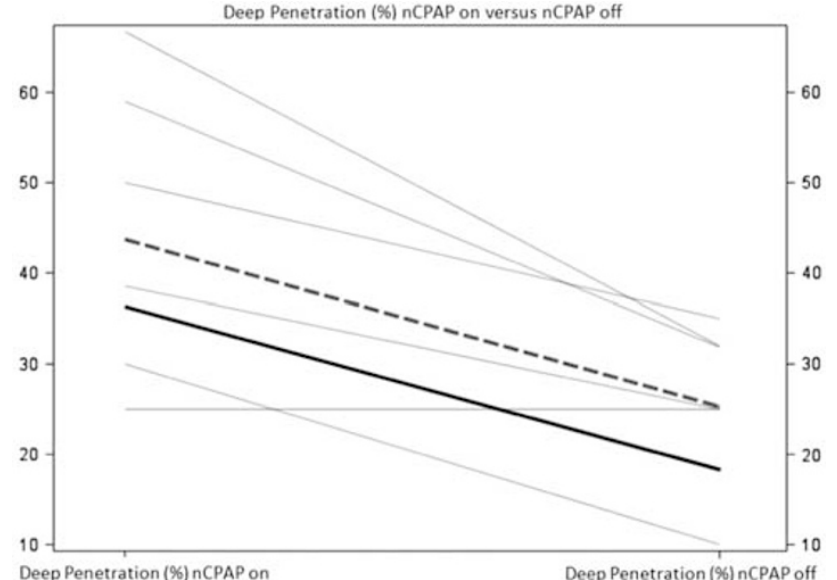

Figure 1. Comparative line graph demonstrating the reduction of deep penetration events (\%) when nCPAP was turned off. The solid lines represent each of the seven participants' percentage of deep penetration from one condition to the other. The dashed line represents the mean percentage of deep penetration for all seven participants, revealing a decreased frequency of deep penetration events from the on-nCPAP condition to the off-nCPAP condition. The bold line represents the full-term infant who participated in the study.

All seven infants enrolled in this study demonstrated swallowing dysfunction during on-nCPAP and were therefore studied offnCPAP. A total of 20 swallows were assessed under each condition: on-nCPAP and off-nCPAP, for a total of 40 analyzed swallows for each participant. Percentages were derived for the occurrence of any swallow dysfunction within each condition (Table 2). Swallowing dysfunction was reported under both conditions, but it was greater during nCPAP administration. Swallowing dysfunction directly affecting airway protection included deep penetration and aspiration, both of which decreased significantly off-nCPAP compared with on-nCPAP (Table 2). This finding is visually expressed for each patient tested in Figures 1 and 2, demonstrating that six of the seven patients had reduced dysfunction while off-nCPAP. Mild penetration $(P=0.656)$ and nasopharyngeal reflux $(P=0.875)$ remained the same when we compared on-nCPAP and off-nCPAP values. Figures $3 a-c$ show still frames from the fluoroscopy study of participant no. 4, demonstrating the effect of nCPAP on swallowing. None of the seven participants had a cough response after each aspiration event, indicating 'silent aspiration'.

The following findings were observed; however, they did not reach statistical significance. The ability of the infants to clear aspirated material out of the trachea with subsequent swallowing was sufficient in 5/7 participants during on-nCPAP versus $4 / 7$ participants during off-nCPAP. After material penetrated the laryngeal vestibule, the material was not observed to sufficiently

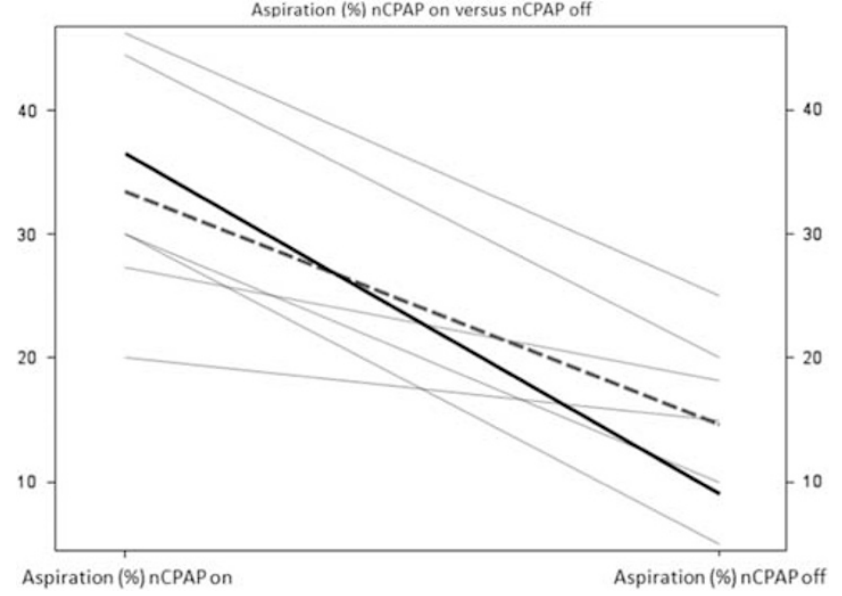

Figure 2. Comparative line graph demonstrating the reduction of aspiration events (\%) when nCPAP was turned off. The solid lines represent each of the seven participants' percentage of aspiration from one condition to the other. The dashed line represents the mean percentage of aspiration for all seven participants, revealing a decreased frequency of aspiration events from the on-nCPAP condition to the off-nCPAP condition. The bold line represents the full-term infant who participated in the study.

clear in 2/7 participants during on-nCPAP, while all participants had good clearance of the laryngeal vestibule during off-nCPAP. Analysis of postswallow residue within the pharynx is demonstrated in Table 3. Participant nos. 2 and 7 showed improvements in pharyngeal clearance from severe to absent and mild to absent, respectively, when nCPAP was not in use.

The observations and rating of the VFSS had good interjudge reliability. Data points assessed by the research team and independent clinician yielded $92 \%$ agreement across all swallows for all seven participants.

\section{DISCUSSION}

We assessed the effects of nCPAP on pharyngeal swallowing in neonates. We hypothesized that nCPAP would increase the risk for airway compromise and aspiration during oral feeding in this group of patients. Our findings demonstrate that nCPAP alters the pharyngeal swallowing mechanism in neonates. Oral feeding while on nCPAP significantly increases the risk of laryngeal penetration and tracheal aspiration events. This provides neonatologists with important information regarding the decision to initiate oral feeding in infants who receive noninvasive ventilation. Based on these results, our NICU at Winthrop University Hospital changed practice and no longer allows oral feeding while infants are on nCPAP. 

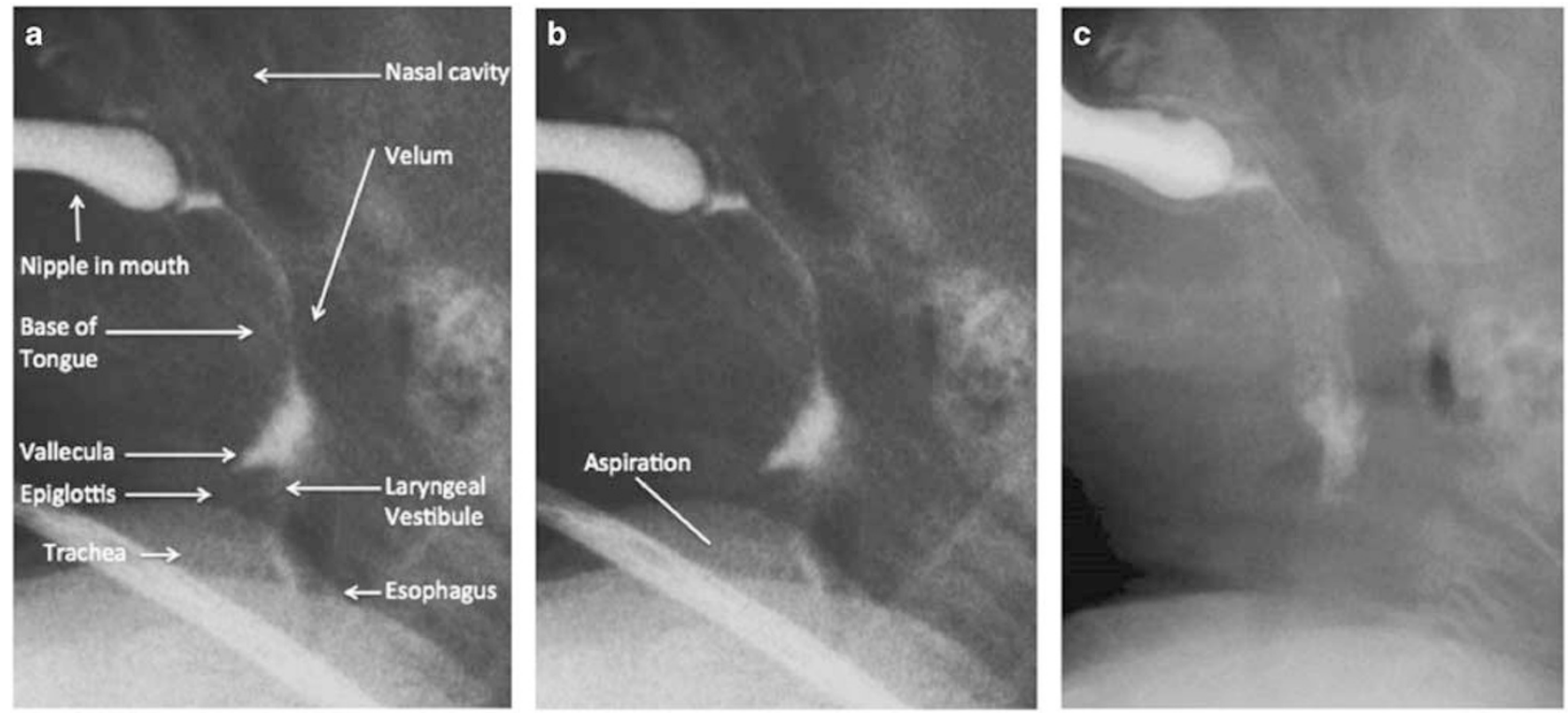

Figure 3. (a) Still frame of participant no. 4, identifying anatomical structures. (b) Still frame of participant no. 4 with nCPAP in use, showing evidence of contrast material within the laryngeal vestibule and a smaller scatter of material within the trachea. (c) The same participant with nCPAP not in use. At the time of the swallow trigger, no contrast material was observed within the laryngeal vestibule or trachea.

Table 3. Presence and severity of pharyngeal residue after swallow

\begin{tabular}{lll}
\hline Participant & on-nCPAP & off-nCPAP \\
\hline 1 & Mild & Mild \\
2 & Severe & Absent \\
3 & Absent & Absent \\
4 & Mild & Mild \\
5 & Mild & Mild \\
6 & Mild & Mild \\
7 & Mild & Absent \\
\hline Abbreviation: nCPAP, nasal continuous positive airway pressure.
\end{tabular}

Initiation of oral feeding represents a serious challenge in NICUs, as neonatologists need to balance the infants' medical status with developmental readiness. The fact that preterm infants are often dependent on nCPAP for several weeks challenges the medical team to decide whether oral feedings should be initiated at the developmentally appropriate age of approximately 34 weeks' corrected gestational age ${ }^{16}$ or to delay the introduction of oral feedings until the infant shows improved respiratory status without the use of respiratory devices.

Delaying the initiation of oral feeding is associated with slower progression to full oral feeding status. ${ }^{17} \mathrm{~A}$ recent study carried out by Hanin et al. ${ }^{18}$ demonstrated that infants with bronchopulmonary dysplasia and were fed orally while on nCPAP were able to fully feed orally compared with infants who were not fed orally while receiving nCPAP. However, the study only used chest $X$-ray to exclude the possibility of aspiration pneumonia, but no assessment was performed to evaluate airway safety or the possibility of micro-aspiration that will not be evidenced by plain chest X-ray. That study was not powered to detect differences in respiratory outcome.

Aspiration in our study was clinically occult. This may account for the lack of recognition that potentially harmful aspiration might occur for babies both on- or off-nCPAP. If undetected, these events may exaggerate a wide spectrum of pulmonary disorders. ${ }^{19}$
The type(s) of complications resulting from aspiration depends on the quantity and nature of the aspirated material, chronicity and host responses. Chronic aspiration has been reported to result in persistent smoldering inflammation, disease progression and eventually chronic lung injury. ${ }^{20,21}$ Specific pulmonary pathologies owing to aspiration reported in the literature include recurrent pneumonias, $^{22}$ bronchospasm, laryngospasm, ${ }^{23}$ fibrosis, decreased functional lung capacity, respiratory failure and death. ${ }^{24}$ NICUs use nasoduodenal and nasojejunal feeding in babies with broncho-pulmonary dysplasia to avoid aspiration and enhance fast recovery. The prevention of aspiration in infants who already require respiratory devices is of significant importance for both short- and long-term lung health.

To our knowledge, no studies have been performed on human infants to evaluate the effect of nCPAP on the pharyngeal swallowing mechanism. Previous studies utilizing neonatal lambs revealed that the application of nCPAP had no deleterious effect on nutritive swallowing-breathing coordination, cardiopulmonary safety, feeding efficiency ${ }^{25}$ or on the esophageal phase of swallowing. ${ }^{26}$ Translation of these results from newborn lambs to human infants must be made with caution, as anatomical and physiological differences between human infants and lambs exist in the aero-digestive tract. Our results, obtained in sick term and preterm infants with altered lung function and respiratory insufficiency, suggest that deleterious effects are associated with oral feeding during application of nCPAP.

In addition to our finding that airway safety is significantly compromised when neonates swallow in the presence of continuous pressurized airflow via nCPAP, a secondary, and surprising, finding was that even in the absence of nCPAP many study participants continued to show airway compromise (as demonstrated in Table 2 and Figures 1 and 2), although it was significantly less frequent than while on nCPAP. We propose three potential causes for this observation: (a) sensory adaptation, (b) prior feeding experiences, and (c) underlying lung disease. First, the theory of sensory adaptation describes an observed decrease in responsiveness to constant stimuli. ${ }^{27}$ Sensory habituation was documented in a study by Dodrill, ${ }^{28}$ who assessed habituation to physical stimulus in preterm infants who required prolonged tube feedings. ${ }^{28,29}$ Applying this theory to our findings, we suggest that 
mechanoreceptors may have remained in a condition of 'habituation' after the nCPAP was turned off and remained that way while we tested the infants in the off-nCPAP condition as well. The second proposed cause for the secondary finding in this study is the infants' feeding experiences. ${ }^{30}$ Bu'Lock et al. ${ }^{31}$ proposed that persistence of immature feeding patterns may be due to maladaptive, learned responses associated with breathing difficulties during feedings. All of our study participants had learned how to feed orally with nCPAP. If the experience of feeding on nCPAP in our study participants had required them to alter their oral pharyngeal movements during feeding, it is probable that, when removed from the nCPAP, they continued to demonstrate previously learned motor patterns. Third, infants may have continued to show swallow dysfunction while in the off-nCPAP condition because of the underlying lung disease. Ample research has proven that preterm infants with chronic lung disease, respiratory distress syndrome and/or broncho-pulmonary dysplasia may have limited respiratory control because of issues such as irregular breathing patterns associated with oral feedings and/or delays in the normal maturation of stable suck, swallow and breathing coordination. ${ }^{32-34}$ To our knowledge, no prospective research studies have used video fluoroscopy to assess the swallowing mechanism of neonates with such significant respiratory requirements. Therefore, we do not know what the baseline is for these infants.

A limitation of this study is the small number of participants. As stated previously, our NICU Safety Committee halted enrollment after interim review of the results of the first seven participants because of the documented risks observed with oral feeding while neonates are on nCPAP. It was considered unethical to expose infants to harm after a clear safety risk was identified. Even though we had only seven patients, our results demonstrated significant differences in the risk of airway compromise associated with oral feeding while on nCPAP.

Our inclusion criteria did not include a specific gestational age requirement. As only participant no. 1 was born full term at 38 weeks; we redid the analysis without that participant; however, the risk of aspiration remained significant on-nCPAP compared with off-nCPAP $(P=0.031)$. After review of both data sets, this participant was included in the final analysis to show transparency in our data and all participants enrolled. Of note, all participants had PMA at the time of the study $\geqslant 37$ weeks, except for one infant (PMA 34.1 weeks).

In our study design, we allowed each infant a 90-s period of time to feed prior to direct visualization. This was provided for two main reasons. First, we allowed the infant to organize or adjust to the feeding prior to assessment and minimize the acclimatization effect. Second, in accord with a previous study, ${ }^{14}$ which revealed that if infants were to demonstrate swallowing dysfunction, it will occur after the first $90 \mathrm{~s}$. These two reasons helped reduce radiation exposure and increased the validity of our findings. The acclimatization effect was further reduced by designing the enrollment criteria to include participants who performed at least $50 \%$ of their enteral feeding by mouth before the study.

Our study is the first to evaluate the effect of nCPAP on pharyngeal swallowing mechanism in neonates. This new information obtained from our study strengthens our understanding regarding the swallowing and breathing acts required by neonates in order to feed safely and successfully by mouth. The observations presented here are the first step toward identifying some of these elements. Further work is required to evaluate the significance of our findings in terms of clinical outcomes and the relationship between swallowing safety and respiratory devices so that evidence-based oral feeding initiation protocols can be established.

\section{CONCLUSION}

Based on our findings, we conclude that oral feeding while on nCPAP may significantly increase the risk of laryngeal penetration and tacheal aspiration events. These events can result in deleterious cardio-respiratory complications, such as progressive chronic lung disease, in preterm infants. We recommend caution when initiating oral feeding on nCPAP. There was also a high rate of aspiration in the group of patients even when off nCPAP. Further research is needed to establish evidence-based oral feeding protocols for this at-risk population.

\section{CONFLICT OF INTEREST}

The authors declare no conflict of interest.

\section{ACKNOWLEDGEMENTS}

We extend our sincere appreciation to all Winthrop NICU nurses who assisted in conducting the study.

\section{REFERENCES}

1 Mahmoud RA, Roehr CC, Schmalisch G. Current methods of non-invasive ventilatory support for neonates. Paediatr Respir Rev 2011; 12: 196-205.

2 Sankaran K, Adegbite M. Non invasive respiratory support in neonates: a brief review. Chinese J Contemp Pediatr 2012; 14(9): 643-665.

3 Gaon P, Lee S, Hannan S, Ingram D, Milner AD. Assessment of effect of nasal continuous positive pressure on laryngeal opening using fiber optic laryngoscopy. Arch Dis Child 1999; 80: F230-F232.

4 Nishino T, Sugimori K, Kohchi A, Hiraga K. Nasal constant positive airway pressure inhibits the swallowing reflex. Am Rev Respir Dis 1989; 140: 1290-1293.

5 Shaker CS. Nipple feeding preterm infants: an individualized, developmentally supportive approach. Neonatal Netw 1999; 18(3): 15-22.

6 Shaker CS. Cue-based co-regulated feeding in the neonatal intensive care unit: supporting parents in learning to feed their preterm infant. Newborn Infant Nurs Rev 2013; 13(1): 51-55.

7 Thoyre SM, Shaker CS, Pridham K. The early feeding skills assessment for preterm infants. Neonatal Netw 2004; 24(3): 7-16.

8 lyer NP, Chatburn R. Evaluation of a nasal cannula in noninvasive ventilation using a lung simulator. Respir Care 2015; 60(4): 508-512.

9 De Paoli AG, Morley C, Davis P, Lau R, Hingeley E. In vitro comparison of nasal continuous positive airway pressure devices for neonates. Arch Dis Child Fetal Neonatal Ed 2002; 87(1): F42-F45

10 Fink TA, Ross JB. Are we testing a true thin liquid? Dysphagia 2009; 24(3): 285-289.

11 Lee JH, Chang YS, Yoo HS, Ahn SY, Seo HJ, Choi SH et al. Swallowing dysfunction in very low birth weight infants with oral feeding desaturation. World J Pediatr 2011; 7(4): 337-343.

12 Seluk B, Uysal H, Aydogdu I, Akyuz M, Ertekin C. Effect of temperature on electrophysiological parameters of swallowing. J Rehabil Res Dev 2007; 44(3): 373.

13 Rogers R, Arvedson J. Assessment of infant oral sensorimotor and swallowing function. Ment Retard Dev Disabil Res Rev 2005; 11(1): 74-82.

14 Newman LA, Keckley C, Petersen MC, Hamner A. Swallowing function and medical diagnoses in infants suspected of dysphagia. Pediatrics 2001; 108(6): e106.

15 Tutor JD, Gosa MM. Dysphagia and aspiration in children. Pediatr Pulmonol 2012; 47(4): 321-337.

16 Breton S, Steinwender S. Timing introduction and transition to oral feeding in preterm infants: current trends and practice. Newborn Infant Nurs Rev 2008; 8(3): 153-159.

17 Simpson C, Schanler RJ, Lau C. Early introduction of oral feeding in preterm infants. Pediatrics 2002; 110(3): 517-522.

18 Hanin M, Nuthakki S, Malkar MB, Jadcherla SR. Safety and efficacy of oral feeding in infants with BPD on nasal CPAP. Dysphagia 2015; 30(2): 121-127.

$19 \mathrm{Hu} \mathrm{X}$, Lee JS, Pianoso PT, Ryu JH. Aspiration-related pulmonary syndromes. Chest 2015; 147(3): 815-823.

20 Radford PJ, Stillwell PC, Blue B, Hertel G. Aspiration complicating bronchopulmonary dysplasia. Chest 1995; 107(1): 185-188.

21 Boesch RP, Wood RE. Aspiration. In: Kendig and Chernick's Disorders of the Respiratory Track in Children, 8th edn. Elsevier: Philadelphia, PA, USA, 2012, pp 947-948.

22 Taniguchi M, Moyer R. Assessment of risk factors for pneumonia in dysphagia children: significance of video- fluoroscopic swallowing evaluation. Dev Med Child Neurol 1994; 36: 495-502. 
23 Smith CH, Logemann JA, Colangelo LA, Rademaker AW, Pauloski BR. Incidence and patient characteristics associated with silent aspiration in the acute care setting. Dysphagia 1999; 14: 1-7.

24 Seddon PC, Khan Y. Respiratory problems in children with neurological impairment. Arch Dis Child 2003; 88(1): 75-78.

25 Bernier A, Catelin C, Ahmed MA, Samson N, Bonneau P, Praud P. Effects of nasal continuous positive-airway pressure on nutritive swallowing in lambs. J Appl Physioly 2012; 112(12): 1984-1991.

26 Djeddi D, Cantin D, Samson N, Tian H, Praud JP. Absence of effect of nasal continuous positive-airway pressure on the esophageal phase of nutritive swallowing in newborn lambs. J Pediatr Gastroenterol Nutr 2013; 57: 188-191.

27 Lawless HT, Heymann H. Sensory Evaluation of Food: Principles and Practices, 1st edn. Chapman and Hall: NewYork, NY, USA, 1998.

28 Dodrill P, McMahon S, Ward E, Weir K, Donovan T, Riddle B. Long-term oral sensitivity and feeding skills of low-risk pre-term infants. Early Hum Dev 2004; 76(1): 23-37.
29 Morris S. Development of oral-motor skills in the neurologically impaired child receiving non-oral feedings. Dysphagia 1989; 3: 135-154.

30 Lau C, Smith EO, Schanler RJ. Coordination of suck-swallow and swallow respiration in preterm infants. Acta Paediatr 2003; 92(6): 721-727.

31 Bu'Lock F, Woolridge MW, Baum JD. Development of coordination of sucking, swallowing and breathing: ultrasound study of term and preterm infants. Dev Med Child Neurol 1990; 32: 669-678.

32 Craig CM, Lee DN, Freer YN, Laing IA. Modulations in breathing patterns during intermittent feeding in term infants and preterm infants with bronchopulmonary dysplasia. Dev Med Child Neurol 1999; 41: 616-624.

33 Gewolb IH, Vice FL, Schwietzer-Kenney EL, Taciak VL, Bosma JF. Developmental patterns of rhythmic suck and swallow in preterm infants. Dev Med Child Neurol 2001; 43(1): 22-27.

34 da Costa SP, van der Schans CP, Zweens MJ, Boelema SR, van der Meij E, Boerman MA et al. Development of sucking patterns in pre-term infants with bronchopulmonary dysplasia. Neonatology 2010; 98(3): 268-277. 